\title{
WAIST OF MAPS MEASURED VIA URYSOHN WIDTH
}

\author{
ALEXEY BALITSKIY* AND ALEKSANDR BERDNIKOV*
}

\begin{abstract}
We discuss various questions of the following kind: for a continuous map $X \rightarrow Y$ from a compact metric space to a simplicial complex, can one guarantee the existence of a fiber large in the sense of Urysohn width? The $d$-width measures how well a space can be approximated by a $d$-dimensional complex. The results of this paper include the following.

(1) Any piecewise linear map $f:[0,1]^{m+2} \rightarrow Y^{m}$ from the unit euclidean $(m+2)$-cube to an $m$-polyhedron must have a fiber of 1 -width at least $\frac{1}{2 \beta m+m^{2}+m+1}$, where $\beta=\sup _{y \in Y} \operatorname{rk} H_{1}\left(f^{-1}(y)\right)$ measures the topological complexity of the map.

(2) There exists a piecewise smooth map $X^{3 m+1} \rightarrow \mathbb{R}^{m}$, with $X$ a riemannian $(3 m+1)$ manifold of large $3 m$-width, and with all fibers being topological $(2 m+1)$-balls of arbitrarily small $(m+1)$-width.
\end{abstract}

\section{INTRODUCTION}

The notion of the Urysohn width of a compact metric space was suggested by Pavel Urysohn in 1920s (and published posthumously by Pavel Alexandrov [3]). The $d$-width measures how well a space can be approximated by a $d$-dimensional simplicial complex. A compact metric space $X$ is said to have $d$-width at most $w$, if there is a continuous map $X \rightarrow Z^{d}$ to a $d$-dimensional simplicial complex with all fibers having diameter at most $w$. The original definition of Urysohn was given in terms of closed coverings, and we give an overview of different equivalent ways of defining width in Section 1 .

The Urysohn width of a riemannian manifold is related to other metric invariants. For example, the codimension 1 width does not exceed the $n^{\text {th }}$ root of the volume (up to a dimensional factor; see [14]), and bounds from above the filling radius of a manifold (see [8, Appendix 1]) and its hypersphericity (see [9, Proposition $\mathrm{F}_{1}$ ] or [11, Section 5]). Among the applications of the Urysohn width we mention a recent transparent proof [21] of Gromov's systolic inequality, building on the ideas from [22, 12].

The question raised in this paper is inspired by another famous Gromov's inequality, namely the waist of the sphere theorem [10]. It says that any generic smooth map $f$ : $S^{n} \rightarrow \mathbb{R}^{m}, m<n$, has a fiber of $(n-m)$-volume at least the one of the $(n-m)$-dimensional "equatorial" subsphere. The target space can be replaced by any $m$-manifold [15, while it is not clear if one can replace it by an $m$-polyhedron $Y^{m}$. The only result in this direction we are aware of is [2, Theorem 7.3], saying that any generic smooth map $S^{n} \rightarrow Y^{n-1}$ has a fiber of length $\geq \pi$. A non-sharp version of the waist theorem, however, can be proved for any $m$-dimensional target space by induction using the Federer-Fleming isoperimetric estimate. This type of argument apparently goes back to Almgren, and it was used by Gromov in [8] (see the exposition in [13, Section 7], which applies to any target space, or in [1, Section 7]). A discrete version of this non-sharp estimate is proven in [20] along the same lines. For Riemannian metrics other than round, the case $n=2$ is understood [18, 4], and the case $n=3$ is investigated under additional curvature assumptions [19].

The Urysohn width itself is a waist-type invariant, in which the size of a fiber is measured via its diameter, instead of the volume. In this paper, we investigate (non-sharp) waist theorems, where the size of a fiber is measured via the Urysohn width. 
Prototype question. Fix integers $n, m, d$, such that $n>m+d$. Let $f: X^{n} \rightarrow Y^{m}$ be a continuous map from a compact riemannian $n$-manifold to an m-dimensional simplicial complex. Let $w$ be the supremal Urysohn d-width of fibers $f^{-1}(y), y \in Y$, viewed as compact metric spaces with the extrinsic metric of $X$. Can one bound $w$ from below in terms of the $(n-1)$-width of $X$ ? If not, can one bound $w$ if the "topological complexity" of the fibers is restricted?

It is natural to expect that the answer should be affirmative in some sense when $n>$ $m+d$ (if we hope that the corresponding property of the dimension [25, 6] is robust). When $d=1$, and the first Betty number of the fibers is bounded, this is indeed the case, as we will show in Section 3 . However, in general this is far from true. In Section 4 it will be shown that even for $n=(m+1)(d-m)+2 m$ and topologically trivial fibers the answer is negative. In a sense, this shows the failure of the notion of the $d$-width to measure the "defect of $d$-dimensionality".

Let us describe the answers for the first four non-trivial cases of Prototype question. These four claims are the simplest special cases of the theorems explained in this paper.

(A) There is a map $f:[0,1]^{3} \rightarrow[0,1]$ with all fibers having arbitrarily small 1-width.

We describe this example $\left(\left[9\right.\right.$, Example $\left.\left.\mathrm{H}_{1}^{\prime \prime}\right]\right)$ briefly. Consider an $\varepsilon$-fine cubical grid in $\mathbb{R}^{3}$, and let $Z_{0}$ be its 1 -skeleton. Let $Z_{1}$ be the 1 -skeleton of the dual grid. Define $f$ by setting $f(x)=\frac{\operatorname{dist}\left(x, Z_{0}\right)}{\operatorname{dist}\left(x, Z_{0}\right)+\operatorname{dist}\left(x, Z_{1}\right)}$. It can be checked that every fiber $\Sigma_{y}=f^{-1}(y), y \in[0,1)$, retracts to $Z_{0}$ with every point moving by distance $\lesssim \varepsilon$; hence it has small 1-width. Similarly, the fibers over $y \in(0,1]$ are approximated by $Z_{1}$.

In Section 2, we explain how this example is generalized to higher dimensions, see Theorem 2.2. This might be known to experts, but we were not able to locate a reference.

(B) Notice that all regular fibers in the previous example have high genus. What happens if we bound their topological complexity?

Suppose that a piecewise linear map $f:[0,1]^{3} \rightarrow[0,1]$ is such that all fibers $f^{-1}(y), y \in[0,1]$, are homeomorphic to $[0,1]^{2}$. Then there is a fiber $f^{-1}(y)$ of Urysohn 1-width at least $\frac{1}{3}$.

This is the baby case of one of our main results, Theorem 3.16. Here is the idea of the proof that will be developed in Section 3. Suppose that every fiber $X_{y}=f^{-1}(y)$ has width $\mathrm{UW}_{d}\left(X_{y}\right)<c$. So there are maps $X_{y} \rightarrow Z_{y}$ to graphs $Z_{y}$ whose fibers are of diameter less than $c$. A naïve idea might be to assemble them together to get a map $[0,1]^{3} \rightarrow \bigcup Z_{y}$. If there was a nice way to interpret $\bigcup Z_{y}$ as a two-dimensional space, then we would be done as long as $c<\mathrm{UW}_{n-1}(X)$. A careful argument might try to assemble the maps $X_{y} \rightarrow Z_{y}$ by induction on the skeletal structure of $Y$, subdivided finely. The newly built intermediate maps will have fibers with the size bounded in terms of $c$ and the "topological complexity" of the $X_{y}$.

(C) The following is a special case of [9, Corollary $\left.\mathrm{H}_{1}^{\prime}\right]$, which we discuss in Section 2 (see Theorem 2.1).

Every continuous map $f: X^{4} \rightarrow Y^{1}$ from a compact metric space to a graph has a fiber whose 1-width is at least the 3 -width of $X$.

(D) Another major result of this paper is Theorem 4.1, a family of examples of maps with small and topologically trivial fibers; here is the simplest case.

There is a riemannian metric on $[0,1]^{4}$ that has substantial 3 -width but the fibers of the coordinate projection $f:[0,1]^{4} \rightarrow[0,1]$ all have small 2-width. 
We sketch roughly the idea of the construction. For each $y \in[0,1]$, the standard metric inside the fiber $f^{-1}(y) \simeq[0,1]^{3}$ is modified as follows. Consider the high-genus surface $\Sigma_{y} \subset f^{-1}(y)$, as in the example(A), In its small tubular neighborhood, blow up the metric in the normal direction; then, squeeze the metric everywhere outside the tubular neighborhood. The result can be mapped to the suspension of $Z_{0}$ or $Z_{1}$ with small fibers. However, the entire space $[0,1]^{4}$ can be shown to have substantial 3-width.

It is worth noting that the theorems explained in this paper do not answer Prototype question completely. For example, imagine one has a map $X^{k^{2}} \rightarrow Y^{k-1}$ with topologically trivial fibers $(k \geq 3)$, and with $\mathrm{UW}_{k^{2}-1}\left(X^{k^{2}}\right)=1$. Theorem 4.1 implies that it might happen that all the fibers have $(2 k-1)$-width arbitrarily small. Theorem 2.1 implies that there is a fiber $F$ with $\mathrm{UW}_{k-1}(F) \geq 1$. It is not clear though what can be said about the widths $\mathrm{UW}_{d}$ of the fibers in the range $k-1<d<2 k-1$.

Acknowledgements. We thank Larry Guth for helpful discussions. This paper arose from a chapter of the thesis defended by the first-named author under the supervision of Larry Guth at MIT.

\section{URYSOHN WIDTH}

Everywhere in this section, $X$ denotes a compact metric space. The diameter of a set is measured using the distance function in $X: \operatorname{diam} A=\sup _{a, a^{\prime} \in A} \operatorname{dist}_{X}\left(a, a^{\prime}\right)$.

Definition 1.1. The Urysohn $d$-width of a closed subset $S$ of a compact metric space $X$ can be defined in either of the following ways.

$$
\mathrm{UW}_{d}(S)=\inf _{\cup U_{i} \supset S} \sup _{i} \operatorname{diam}\left(U_{i}\right)
$$

where the infimum is taken over all open covers of $S$ of multiplicity at most $d+1$.

$$
\mathrm{UW}_{d}(S)=\inf _{\cup C_{i}=S} \sup _{i} \operatorname{diam}\left(C_{i}\right)
$$

where the infimum is taken over all finite closed covers of $S$ of multiplicity at most $d+1$.

$$
\mathrm{UW}_{d}(S)=\inf _{p: S \rightarrow Z} \sup _{z \in Z} \operatorname{diam}\left(p^{-1}(z)\right)
$$

where the infimum is taken over all continuous maps $p$ from $S$ to any metrizable topological space $Z$ of covering dimension at most $d$.

The quantity $\mathrm{W}(p)=\sup _{z \in Z} \operatorname{diam}\left(p^{-1}(z)\right)$ will be called the width of the map $p$.

The class of test spaces $Z$ in $(\overline{\mathrm{UM}})$ can be narrowed down to $d$-dimensional simplicial complexes, without changing the width, as it will implicitly follow from the proof below.

Proof of the equivalence of different definitions of the Urysohn width.

Denote by $w_{\mathrm{c}}, w_{\mathrm{o}}, w_{\mathrm{m}}$ the width of a set $S \subset X$ measured as in (UC), (UO), (UM), respectively.

UO $\leq \mathrm{UC}$ Given a finite closed covering $S=\bigcup C_{i}$, we can use compactness to argue that

$$
\delta_{i_{0}, \ldots, i_{d+1}}=\min _{x \in X} \max _{0 \leq k \leq d+1} \operatorname{dist}\left(x, C_{i_{k}}\right)
$$

is attained and positive. Take $\varepsilon>0$ smaller then each $\delta_{i_{0}, \ldots, i_{d+1}}$ over all collections of indices $i_{0}<\ldots<i_{d+1}$, and consider the open covering $\left\{U_{i}\right\}$, where $U_{i}=U_{\varepsilon}\left(C_{i}\right)$ is the $\varepsilon$-neighborhood of $C_{i}$. It has the same multiplicity as the covering $\left\{C_{i}\right\}$, and max diam $U_{i} \leq \max \operatorname{diam} C_{i}+2 \varepsilon$. Taking $\varepsilon \rightarrow 0$, we get $w_{\mathrm{o}} \leq \max \operatorname{diam} C_{i}$. Therefore, $w_{\mathrm{o}} \leq w_{\mathrm{c}}$. 
$(\overline{\mathrm{UC}} \leq \mathrm{UM})$ Suppose we are given a map $p: S \rightarrow Z^{d}$ to a metrizable space; fix a metric on $Z$. Recall that the width of $p$ is defined as $\mathrm{W}(p)=\sup _{z \in Z} \operatorname{diam}\left(p^{-1}(z)\right)$. Fix a small number $\varepsilon>0$. For each point $z \in p(S)$ one can find radius $r(z)>0$ such that the preimage of $V_{r(z)}(z)$, the $r(z)$-neighborhood of $z$, has diameter smaller than $\mathrm{W}(p)+\varepsilon$. Here we used

$$
\lim _{r \rightarrow 0} \operatorname{diam}\left(p^{-1}\left(V_{r}(z)\right)\right)=\operatorname{diam}\left(p^{-1}(z)\right) .
$$

By definition of dimension (and compactness), there is a finite open covering $\left\{V_{i}\right\}$ of $p(S)$, refining $\left\{V_{r(z)}(z)\right\}$, and with multiplicity at most $d+1$. It follows from Lebesgue's number lemma that there is a closed covering $\left\{D_{i}\right\}$ with $D_{i} \subset V_{i}$. Then the closed sets $C_{i}=p^{-1}\left(D_{i}\right)$ have diameter less than $\mathrm{W}(p)+\varepsilon$, and cover $S$ with multiplicity at most $d+1$. Repeating this with arbitrarily small $\varepsilon$, one gets $w_{\mathrm{c}} \leq \mathrm{W}(p)$. Since this is true for all $p$, we conclude $w_{\mathrm{c}} \leq w_{\mathrm{m}}$.

$\left(\mathrm{UM} \leq \mathrm{UO}\right.$ Given an open covering $S \subset \bigcup U_{i}$ (which we can assume finite by compactness) with multiplicity $d+1$, consider the mapping to its nerve

$$
\varphi: S \rightarrow N^{d}
$$

associated to any subordinate partition of unity. The preimage of every point is entirely contained in some $U_{i}$, hence $\mathrm{W}(\varphi) \leq \sup \operatorname{diam} U_{i}$. Therefore, $w_{\mathrm{m}} \leq w_{\mathrm{o}}$.

Definition 1.1 was given for a closed set $S$. We adopt the following convention: the width of a (not necessarily closed) set $S \subset X$ is defined in terms of open coverings, (UO).

Lemma 1.2. Let $f: X \rightarrow Y$ be a continuous map from a compact metric space $X$ to a metrizable topological space $Y$. The function

$$
y \mapsto \mathrm{UW}_{d}\left(f^{-1}(y)\right)
$$

is upper semi-continuous for any d. Namely,

$$
\mathrm{UW}_{d}\left(f^{-1}(y)\right) \geq \limsup _{y^{\prime} \rightarrow y} \mathrm{UW}_{d}\left(f^{-1}\left(y^{\prime}\right)\right) \text {. }
$$

Proof. If a fiber $f^{-1}(y)$ is covered by open sets $U_{i} \subset X$, with diameters $<\mathrm{UW}_{d}\left(f^{-1}(y)\right)+\varepsilon$ and multiplicity at most $d+1$, then these open sets in fact cover neighboring fibers $f^{-1}\left(y^{\prime}\right)$ as well.

\section{WAIST OF MAPS WITH ARBITRARY FIBERS}

Theorem 2.1 ([9, Corollary $\left.\left.\mathrm{H}_{1}^{\prime}\right]\right)$. Let $X$ be a compact metric space, and let $Y$ be a metrizable topological space of covering dimension $m$. Every continuous map $f: X \rightarrow Y$ has a fiber $f^{-1}(y)$ of $d$-width $\mathrm{UW}_{d}\left(f^{-1}(y)\right) \geq \mathrm{UW}_{n-1}(X)$, where $n=(m+1)(d+1)$.

Proof. The assumptions on $Y^{m}$ imply that $\mathrm{UW}_{d}\left(f^{-1}(y)\right)=\inf _{\text {open } V \ni y} \mathrm{UW}_{d}\left(f^{-1}(V)\right)$. Supposing the contrary to the statement of the theorem, and pulling back a fine open cover of $Y$, we obtain an open cover $\left\{U_{i}\right\}$ of $X$ of multiplicity at most $m+1$, such that $\mathrm{UW}_{d}\left(U_{i}\right)<u:=\mathrm{UW}_{n-1}(X)$ for all $i$. It follows from the definition of the $d$-width that every $U_{i}$ admits an open cover $U_{i}=\bigcup_{j} U_{i j}$ of multiplicity at most $d+1$, with $\operatorname{diam} U_{i j}<u$.

The cover $\left\{U_{i j}\right\}$ of $X$ has multiplicity at most $(m+1)(d+1)$, and it can be assumed finite (by compactness), so we get $\mathrm{UW}_{n-1}(X)<u$, which is absurd.

The relation between dimensions $n, m, d$ in Theorem 2.1 is optimal, as the following result (generalizing example (A) from the introduction) shows. 
Theorem 2.2. Let $n=(m+1)(d+1)-1$, and let $\varepsilon>0$ be any small number. There exists a continuous map $f: B^{n} \rightarrow \triangle^{m}$ from the unit euclidean $n$-ball to the $m$-simplex, whose fibers all have Urysohn d-width less than $\varepsilon$.

Remark 2.3. It is easy to show that $\mathrm{UW}_{n-1}\left(B^{n}\right)>0$. This can be deduced from the Lebesgue covering theorem [17, 7], or from the Knaster-Kuratowski-Mazurkiewicz theorem [16]. In fact, the exact value $\mathrm{UW}_{n-1}\left(B^{n}\right)=\sqrt{\frac{2 n+2}{n}}$ is known (see [24, pp. 84$85,268]$ or [2, Remark 6.10]).

The crucial tool used in the proof of Theorem 2.2 is the local join representation of $\mathbb{R}^{n}$, which will be also used in Section 4 .

Lemma 2.4 (cf. [5, Lemma 4.1]). Fix $\varepsilon>0$. There is a locally finite triangulation of $\mathbb{R}^{n}$ by simplices of diameter $<\varepsilon$, admitting a nice coloring: the vertices receive colors $0,1, \ldots, n$ so that each simplex receives all distinct colors.

Proof. In fact, there is such a triangulation with simplices congruent to one another, via the reflection in the facets. Such a triangulation can be obtained from the type $A$ root system and the corresponding affine Coxeter hyperplane arrangement (see [23, Chapter 6]). (Of course, simpler constructions are also possible.)

Definition 2.5 (cf. [5, Definition 4.2]). Let $n=(m+1)(d+1)-1$, and triangulate $\mathbb{R}^{n}$ by $\varepsilon$-small simplices, as in Lemma 2.4. Define $Z_{i}, 0 \leq i \leq m$, to be the union of all simplices of the triangulation colored by colors $(d+1) i$ through $(d+1) i+d$. We say that $\mathbb{R}^{n}$ is the $\varepsilon$-local join of $d$-dimensional complexes $Z_{0}, \ldots, Z_{m}$.

The name is justified by the following observation: every (top-dimensional) simplex $\sigma$ of the triangulation can be written as the join $\left(\sigma \cap Z_{0}\right) * \ldots *\left(\sigma \cap Z_{m}\right)$; that is, any point $x \in \sigma$ can be written as

$$
x=\sum_{i=0}^{m} t_{i} z_{i}, \quad \text { where } z_{i} \in \sigma \cap Z_{i}, t_{i} \geq 0, \sum_{i=0}^{m} t_{i}=1 .
$$

The coefficients $t_{i}$ are determined uniquely, giving a well-defined join map

$$
\tau: \mathbb{R}^{n} \rightarrow \triangle^{m}=\left\{\left(t_{0}, \ldots, t_{m}\right) \mid t_{i} \geq 0, \sum_{i=0}^{m} t_{i}=1\right\} .
$$

Note that $Z_{i}=\tau^{-1}\left(v_{i}\right)$, where $v_{0}, \ldots, v_{m}$ are the vertices of $\triangle^{m}$. For each vertex $v_{i}$, denote the opposite facet of $\triangle^{m}$ by $v_{i}^{\vee}$. For each complex $Z_{i}$, its $d u a l(m d+m-1)$-dimensional complex is given by $Z_{i}^{\vee}=\tau^{-1}\left(v_{i}^{\vee}\right)$. There are natural retractions

$$
\pi_{i}: \mathbb{R}^{n} \backslash Z_{i}^{\vee} \rightarrow Z_{i}
$$

defined by sending $x=\sum_{i=0}^{m} t_{i} z_{i} \in \sigma$ to $z_{i} \in \sigma \cap Z_{i}$; they are well-defined since $t_{i} \neq 0$ whenever $x \notin Z_{i}^{\vee}$. Note that $\pi_{i}$ moves each point by distance $<\varepsilon$.

Proof of Theorem 2.2. Represent $\mathbb{R}^{n}$ as the $\varepsilon / 2$-local join of $d$-dimensional complexes $Z_{0}, \ldots, Z_{m}$; let $\tau: \mathbb{R}^{n} \rightarrow \triangle^{m}$ be its join map. Take $f$ to be the restriction of $\tau$ on the unit ball $B^{n}$. Let us check that the $d$-width of any fiber $F=f^{-1}\left(t_{0}, \ldots, t_{m}\right)$ is small. Fix any $i$ for which $t_{i} \neq 0$. The (restricted) retraction map $\left.\pi_{i}\right|_{F}: F \rightarrow Z_{i}$ has fibers of diameter $<\varepsilon$, so we are done. 


\section{WAIST OF MAPS WITH FIBERS OF BOUNDED COMPLEXITY}

This section generalizes example (B) from the introduction. The main result, Theorem 3.16, in particular implies the following waist inequality.

Any piecewise linear map $f: X^{m+2} \rightarrow Y^{m}$ from a metric $(m+2)$-polyhedron to an $m$ polyhedron must have a fiber of 1 -width at least $\frac{\mathrm{UW}_{m+1}(X)}{2 \beta m+m^{2}+m+1}$, where $\beta=\sup _{y \in Y} \operatorname{rk} H_{1}\left(f^{-1}(y)\right)$ measures the topological complexity of the map.

3.1. PL maps of polyhedra. We use the word polyhedron to refer to a topological space admitting a structure of a finite simplicial complex (together with rectilinear structure on each simplex), though we do not usually specify this structure. We say a continuous map $X \rightarrow Y$ of polyhedra is a piecewise linear map, or a $P L$ map, if it is simplicial for some fine simplicial structures on $X$ and $Y$.

A polyhedron with a metric space structure (giving the same topology) will be called a metric polyhedron. For example, it could be a polyhedron endowed with a smooth riemannian metric on each maximal simplex, so that the metrics on adjacent simplices match in restriction to their common face.

For a map $f: X \rightarrow Y$, we sometimes denote the preimage $f^{-1}(A)$ of a subset $A \subset Y$ by $X_{A}$, if there is no confusion and $f$ is understood from the context. If $X$ and $A \subset Y$ are polyhedra, and $f$ is a $\mathrm{PL}$ map, then $X_{A}$ is naturally a polyhedron. If additionally $X$ is metric, then $X_{A}$ is metric as well (with the extrinsic metric).

Definition 3.1. We measure the topological complexity using the first Betty number. For a space $X$, we set $\operatorname{tc}(X)=\operatorname{rk} H_{1}(X ; \mathbb{Z})=\operatorname{dim} H_{1}(X ; \mathbb{Q})$. For a map $f: X \rightarrow Y$, we set $\operatorname{tc}(f)=\sup _{y \in Y} \operatorname{tc}\left(X_{y}\right)$.

Remark 3.2. In fact, the estimates 3.11, 3.15, 3.16, 3.17 of this section hold in a stronger form, with $\operatorname{tc}(\cdot)$ replaced by a smaller quantity. Namely, we define $\operatorname{tc}^{\prime}(X)$ as the largest number of linearly independent classes in $H^{1}(X ; \mathbb{Q})$ with pairwise zero cup-products. Similarly, for a map $f: X \rightarrow Y$, we set $\operatorname{tc}^{\prime}(f)=\sup _{y \in Y} \operatorname{tc}^{\prime}\left(X_{y}\right)$. We formulate our results with $\operatorname{tc}(\cdot)$ for simplicity, but in the proofs we indicate the adjustments needed if we use $\operatorname{tc}^{\prime}(\cdot)$.

Example 3.3. If $X$ is a closed connected oriented surface of genus $g$, then $\operatorname{tc}(X)=2 g$ while $\operatorname{tc}^{\prime}(X)=g$. If $X$ is a connected oriented surface of genus $g$ with $q>0$ punctures, then $\operatorname{tc}(X)=\operatorname{tc}^{\prime}(X)=2 g+q-1$.

Lemma 3.4. Every PL map $f: X \rightarrow Y$ of polyhedra satisfies the following regularity assumption. Fix a simplicial structure on $Y$ for which $f$ is simplicial. Fix a simplex $\triangle \subset Y$ (of any dimension), and let $\triangle$ be its relative interior. Then one can pick a $P L$ map $\Psi_{\triangle}: \triangle \times \Sigma_{\triangle} \rightarrow X_{\triangle}$, for some polyhedron $\Sigma_{\triangle}$, such that

- $\Psi_{\triangle}$ is fibered over $\triangle$ :

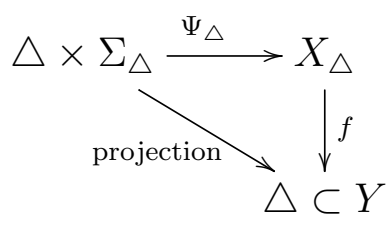

- the restriction

$$
\left.\Psi_{\triangle}\right|_{\triangle \times \Sigma_{\Delta}}: \stackrel{\triangle}{\triangle} \times \Sigma_{\triangle} \rightarrow X_{\triangle}
$$

is a homeomorphism making $f$ a fiber bundle over $\triangle$. 
Proof. For $\Sigma_{\triangle}$, take the fiber over the center of $\triangle$, and the rest can be verified easily.

\subsection{Connected maps.}

Definition 3.5. Let $f: X \rightarrow Y$ be a continuous map of topological spaces. It is called connected if the fibers $f^{-1}(z), z \in Z$, are (nonempty and) path-connected. Every map $f$, connected or not, can be factored as

$$
X \stackrel{\tilde{f}}{\rightarrow} \widetilde{Y} \rightarrow Y
$$

with $\tilde{f}$ connected, and with $\tilde{Y}$ being the space of path-connected components of the fibers of $f$ (topologized by the finest topology making $\widetilde{f}$ continuous). The map $\widetilde{f}$ is called the associated connected map.

If $f$ is a PL map of polyhedra, then $\widetilde{f}$ is also $\mathrm{PL}$, and $\widetilde{Y}$ is a polyhedron having the same dimension as $f(X)$.

Lemma 3.6. Let $f: X \rightarrow Y$ be a connected $P L$ map of polyhedra. If $Y$ is connected then $X$ is connected.

Proof. Let $\gamma:[0,1] \rightarrow Y$ be a path in the base. Fix a simplicial structure of $Y$ for which $f$ is simplicial. Let us build a path $\widetilde{\gamma}:[0,1] \rightarrow X$ covering $\gamma$ in the following weak sense: there is a monotone reparametrization map $r:[0,1] \rightarrow[0,1]$ such that $f(\widetilde{\gamma}(t))=\gamma(r(t))$. First, split $\gamma$ into arcs each of which belongs to a single cell of $Y$. Without loss of generality, there are finitely many of these arcs (this can be achieved by homotoping $\gamma$ slightly, while fixing endpoints). For each such arc $\left[t^{\prime}, t^{\prime \prime}\right] \rightarrow Y$, one can lift $\gamma$ by Lemma 3.4. If $\gamma$ is lifted independently over $\left[t^{\prime}, t\right]$ and $\left[t, t^{\prime \prime}\right]$, the two lifted patches can be connected inside the fiber $f^{-1}(\gamma(t))$. This is how $\widetilde{\gamma}$ can be built. For the assertion of the lemma, having two points $x, x^{\prime} \in X$, one can connect $f(x)$ to $f\left(x^{\prime}\right)$ in the base, and lift the path as above. The endpoints of the lifted path can be connected to $x$ and $x^{\prime}$ in the corresponding fibers. This proves that $X$ is connected.

\subsection{Foliations.}

Definition 3.7. Let $\Sigma$ be a topological space. We use the word foliation to denote a continuous map $p: \Sigma \rightarrow Z$ to a graph (finite 1-dimensional simplicial complex), in the sense that $\Sigma$ is foliated by the fibers $p^{-1}(z), z \in Z$ (the leaves).

This is a non-standard use of the word "foliation". We could have used the word "slicing" as well in this context.

Definition 3.8. Let $\Sigma$ be a polyhedron. We say a foliation $p: \Sigma \rightarrow Z$ is simple if it is a connected PL map.

For a foliation $p$ of a compact metric space $\Sigma$, recall the notation $\mathrm{W}(p)=\sup _{z \in Z} \operatorname{diam} p^{-1}(z)$ for its width. The next lemma shows that, in a sense, any its foliation of width $<1$ can be "simplified" while keeping its width $<1$.

Lemma 3.9. If a metric polyhedron $\Sigma$ admits a foliation of width $<1$, then it also admits a simple foliation width $<1$.

Proof. Let $p: \Sigma \rightarrow Z$ be a foliation of width $<1$. Subdivide $Z$ finely so that the preimage of the open star ${ }^{1} S_{v}$ of every vertex $v \in Z$ has diameter $<1$. Use the simplicial

\footnotetext{
${ }^{1}$ The open star of a vertex of a simplicial complex is the union of the relative interiors of all faces containing the given vertex. In a graph, the open star of a vertex is the vertex itself together with all incident open edges.
} 
approximation theorem to approximate $p$ by a simplicial (for some subdivision of $\Sigma$ ) map $p^{\prime}$ such that for each $x \in \Sigma, p^{\prime}(x)$ belongs to the minimal closed cell of $Z$ containing $p(x)$. It follows that each fiber of $p^{\prime}$ is contained in $p^{-1}\left(S_{v}\right)$ for some $v \in Z$, so $p^{\prime}$ has width $<1$.

Next, replacing $p^{\prime}$ by the associated connected map $\widetilde{p}$ (which is also PL), we arrive at the situation where the leaves $\left(\vec{p}^{\prime}\right)^{-1}(z)$ are (nonempty and) connected for all $z \in Z$, and have diameter $<1$.

\subsection{Interpolation lemma.}

Definition 3.10. Let $\Sigma$ be a topological space, and let $p_{0}: \Sigma \rightarrow Z_{0}, p_{1}: \Sigma \rightarrow Z_{1}$ be its foliations. An interpolation between these is a family of foliations $p_{t}: \Sigma \rightarrow Z_{t}, t \in[0,1]$, continuous in the following sense.

- There is a 2-dimensional polyhedron $Z_{[0,1]}$ together with a parametrization map $\pi: Z_{[0,1]} \rightarrow[0,1]$, such that $\pi^{-1}(t)=Z_{t} \subset Z_{[0,1]}$.

- There is a continuous map $P:[0,1] \times \Sigma \rightarrow Z_{[0,1]}$ fibered over $[0,1]$, and giving $p_{t}$ when restricted over $\{t\}$ :
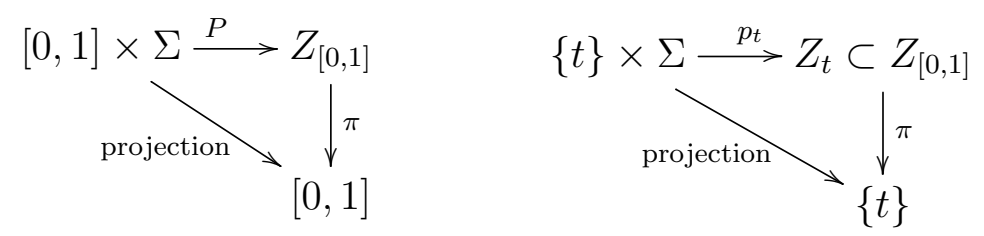

Lemma 3.11. Let $\Sigma$ be a metric polyhedron of topological complexity $\beta=\operatorname{tc}(\Sigma)$, and let $p_{0}: \Sigma \rightarrow Z_{0}, p_{1}: \Sigma \rightarrow Z_{1}$ be simple foliations. It is possible to interpolate between them through simple foliations of width at most $(\beta+2) \mathrm{W}\left(p_{0}\right)+(\beta+1) \mathrm{W}\left(p_{1}\right)$.

We only outline the proof, since a more general statement will be proved in the next subsection. However, this outline illustrates the main method of this section.

Lemma 3.12. Given a (finite) connected graph $Z$ (viewed as a topological space), there is a filtration by closed subspaces $Z^{(t)} \subset Z, t \in[0,1]$, such that

- $Z^{(t)}=\alpha^{-1}([0, t])$, for some continuous function $\alpha: Z \rightarrow[1 / 2,1]$;

- $Z^{(1 / 2)}=\alpha^{-1}(1 / 2)$ consists of a single point;

- every preimage $\alpha^{-1}(t), t \in[1 / 2,1]$, consists of finitely many points (informally, this condition says that $Z^{(t)}$ depends continuously on $\left.t\right)$.

One can also consider a satellite filtration by open subspaces $\check{Z}^{(t)}=\bigcup_{t^{\prime} \in[0, t)} Z^{\left(t^{\prime}\right)}=\alpha^{-1}([0, t))$.

Proof. Such a filtration can be constructed using

$$
\alpha(z)=\frac{\operatorname{dist}_{Z}\left(z_{0}, z\right)}{2 \sup _{z^{\prime} \in Z} \operatorname{dist}_{Z}\left(z_{0}, z^{\prime}\right)}+1 / 2
$$

for any fixed point $z_{0} \in Z$ and any metrization of $Z$.

Outline of the proof of Lemma 3.11. We can assume $\Sigma$ connected (by dealing with each connected component separately).

The graph $Z_{1}$ is connected, since $\Sigma$ is connected, and $p_{1}$ is simple (hence surjective). Filter $Z_{1}$ as in Lemma 3.12 $Z_{1}^{(0)} \subset \ldots \subset Z_{1}^{(t)} \subset \ldots \subset Z_{1}^{(1)}, t \in[0,1]$. We interpolate between $p_{0}$ and $p_{1}$ through foliations $p_{t}: \Sigma \rightarrow Z_{t}$, which can be roughly described as follows. To get a picture of $p_{t}$, first you draw the fibers of $p_{1}$ over $Z_{1}^{(t)}$. Then in the remaining room we draw the fibers of $p_{0}$ (their parts that fit). The resulting picture is interpreted as a foliation by connected leaves, and we call it $p_{t}$ (see Figure 1). 

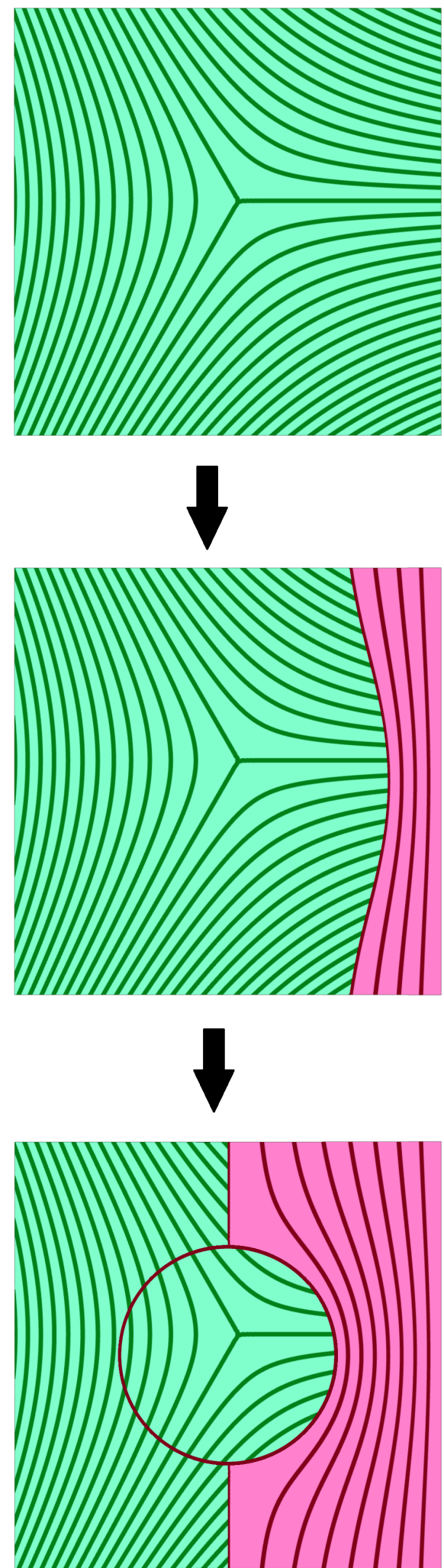
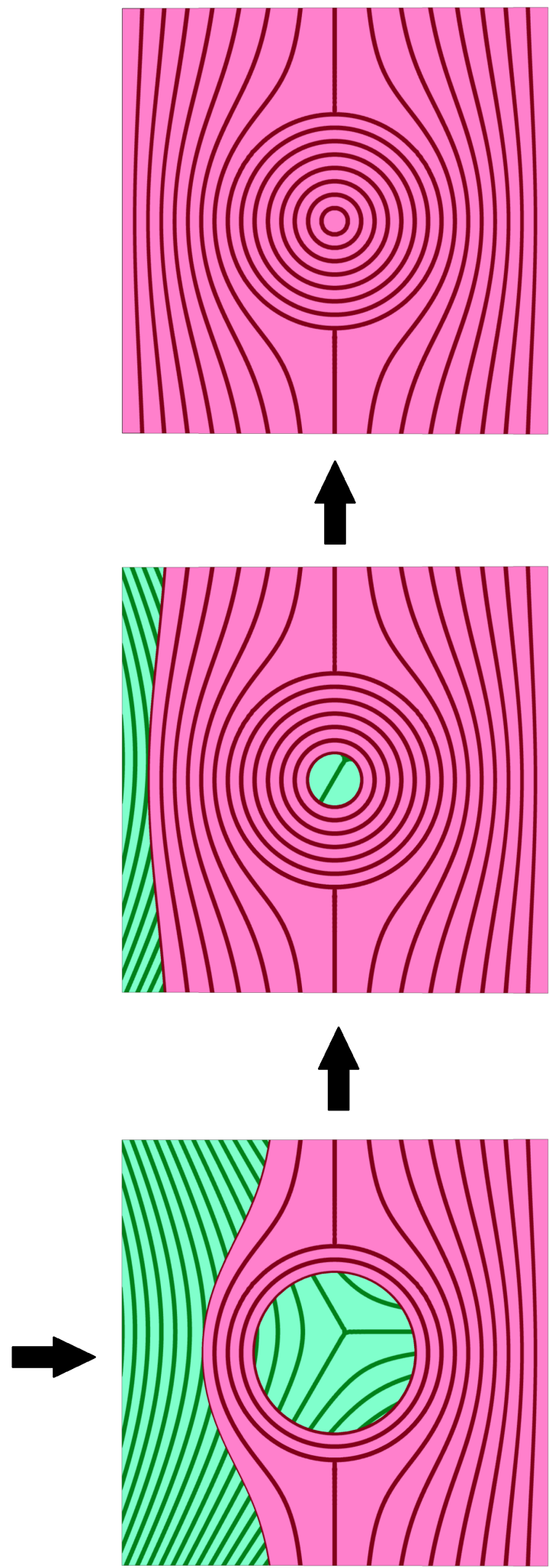

FIgURE 1. Interpolation between foliations. Each rectangle represents a foliation of $\Sigma$, given by a map to a graph. The foliations $p_{0}$ and $p_{1}$ are pictured in green and red, respectively

Let us rigorously describe the space of leaves $Z_{t}$ and the foliation map $p_{t}$. 
- Define $Z_{0}^{(t)}, t \in[0,1]$, as the minimal closed subspace of $Z_{0}$ such that $p_{0}^{-1}\left(Z_{0}^{(t)}\right) \cup$ $p_{1}^{-1}\left(\dot{Z}_{1}^{(t)}\right)=\Sigma$; in other words,

$$
Z_{0}^{(t)}=p_{0}\left(\Sigma \backslash p_{1}^{-1}\left(\dot{Z}_{1}^{(t)}\right)\right)
$$

We write $\Sigma^{(t)}=\Sigma \backslash p_{1}^{-1}\left(\dot{Z}_{1}^{(t)}\right)$ for short.

- The map $\left.p_{0}\right|_{\Sigma^{(t)}}: \Sigma^{(t)} \rightarrow Z_{0}^{(t)}$ might not have all fibers connected, so we factor it through its associated connected map:

$$
\Sigma^{(t)} \stackrel{\widetilde{p}_{0}^{(t)}}{\rightarrow} \widetilde{Z}_{0}^{(t)} \rightarrow Z_{0}^{(t)}
$$

- The graph $Z_{t}$ is defined as

$$
\left(\widetilde{Z}_{0}^{(t)} \sqcup Z_{1}^{(t)}\right) / \stackrel{t}{\sim}
$$

where $\stackrel{t}{\sim}$ is the following equivalence relation. Let us write $z \stackrel{t}{\approx} z^{\prime}$ if $z \in \widetilde{Z}_{0}^{(t)}$, $z^{\prime} \in Z_{1}^{(t)}$, and $\left(\widetilde{p}_{0}^{(t)}\right)^{-1}(z)$ intersects $p_{1}^{-1}\left(z^{\prime}\right)$. Define $\stackrel{t}{\sim}$ to be the transitive closure of $\stackrel{t}{\approx}$. There are natural maps $\iota_{0}^{(t)}: \widetilde{Z}_{0}^{(t)} \rightarrow Z_{t}$ and $\iota_{1}^{(t)}: Z_{1}^{(t)} \rightarrow Z_{t}$.

- The map $p_{t}: \Sigma \rightarrow Z_{t}$ is defined as

$$
p_{t}(x)= \begin{cases}\iota_{1}^{(t)}\left(p_{1}(x)\right), & \text { if } p_{1}(x) \in Z_{1}^{(t)} \\ \iota_{0}^{(t)}\left(\widetilde{p}_{0}^{(t)}(x)\right), & \text { otherwise. }\end{cases}
$$

Observe that for $t=0,1$ this agrees with the original foliations $p_{0}$ and $p_{1}$.

This describes the intermediate foliations $p_{t}$, but in order to describe the interpolation completely we also need to explain how the graphs $Z_{t}$ assemble into a 2-complex $Z_{[0,1]}$, and how the maps $p_{t}$ assemble into a continuous map $P:[0,1] \times \Sigma \rightarrow Z_{[0,1]}$. We do not give these details here, because a more general construction will be explained in the next subsection.

To finish the proof, we need to bound the size of the fibers of $p_{t}$. Why could it be possibly large? Because in the process of interpolating some vertices of the target graph merged under the $\stackrel{t}{\sim}$-identification, so multiple fibers of $p_{0}$ and $p_{1}$ might have been united. Consider a fiber of $p_{t}$. For this fiber, consider the longest chain of identifications

$$
z_{0} \stackrel{t}{\approx} z_{1}^{\prime} \stackrel{t}{\approx} z_{1} \stackrel{t}{\approx} z_{2}^{\prime} \stackrel{t}{\approx} \ldots
$$

with $z_{j} \in \widetilde{Z}_{0}^{(t)}$, and with $z_{j}^{\prime} \in Z_{1}^{(t)}$ all distinct. Suppose it has more than $1+\operatorname{tc}(\Sigma)$ elements of $Z_{1}^{(t)}$. To every subchain $z_{j}^{\prime} \stackrel{t}{\approx} z_{j} \stackrel{t}{\approx} z_{j+1}^{\prime}$ assign an arc inside $\left(\widetilde{p}_{0}^{(t)}\right)^{-1}\left(z_{j}\right)$ connecting some two points $x \in p_{1}^{-1}\left(z_{j}^{\prime}\right)$ and $y \in p_{1}^{-1}\left(z_{j+1}^{\prime}\right)$ (see Figure 22). This arc represents an element of relative homology $H_{1}\left(\Sigma, \Sigma_{1}\right)$, where we denoted $\Sigma_{1}=p_{1}^{-1}\left(Z_{1}^{(t)}\right)$.

Recall that $\Sigma_{1}$ is connected by Lemma 3.6, so $\operatorname{rk} H_{1}\left(\Sigma, \Sigma_{1}\right) \leq \operatorname{tc}(\Sigma)$. There must be a relation between the classes of those arcs in $H_{1}\left(\Sigma, \Sigma_{1}\right)$. It follows that some $z_{j}^{\prime}$ repeats in the chain, which proves that such a chain has at most $1+\operatorname{tc}(\Sigma)$ elements of $Z_{1}^{(t)}$, hence at most $2+\operatorname{tc}(\Sigma)$ elements of $\widetilde{Z}_{0}^{(t)}$. We conclude that the diameter of a fiber of $p_{t}$ is at most $(\beta+2) \mathrm{W}\left(p_{0}\right)+(\beta+1) \mathrm{W}\left(p_{1}\right)$. This finishes the proof if we measure the topological complexity with $\operatorname{tc}(\cdot)$. For the modified complexity $\operatorname{tc}^{\prime}(\cdot)$, one can assign a class in $H^{1}(\Sigma)$ to each element $z_{j}^{\prime}$ (represented by the cochain that counts intersections with $p_{1}^{-1}\left(z_{j}^{\prime}\right)$ ). One needs to verify that there is just one linear dependence between them (coming from the 0-cochain equal to the characteristic function of $\Sigma_{1}$ ), and that their products vanish; this will imply that some $z_{j}^{\prime}$ must repeat. 


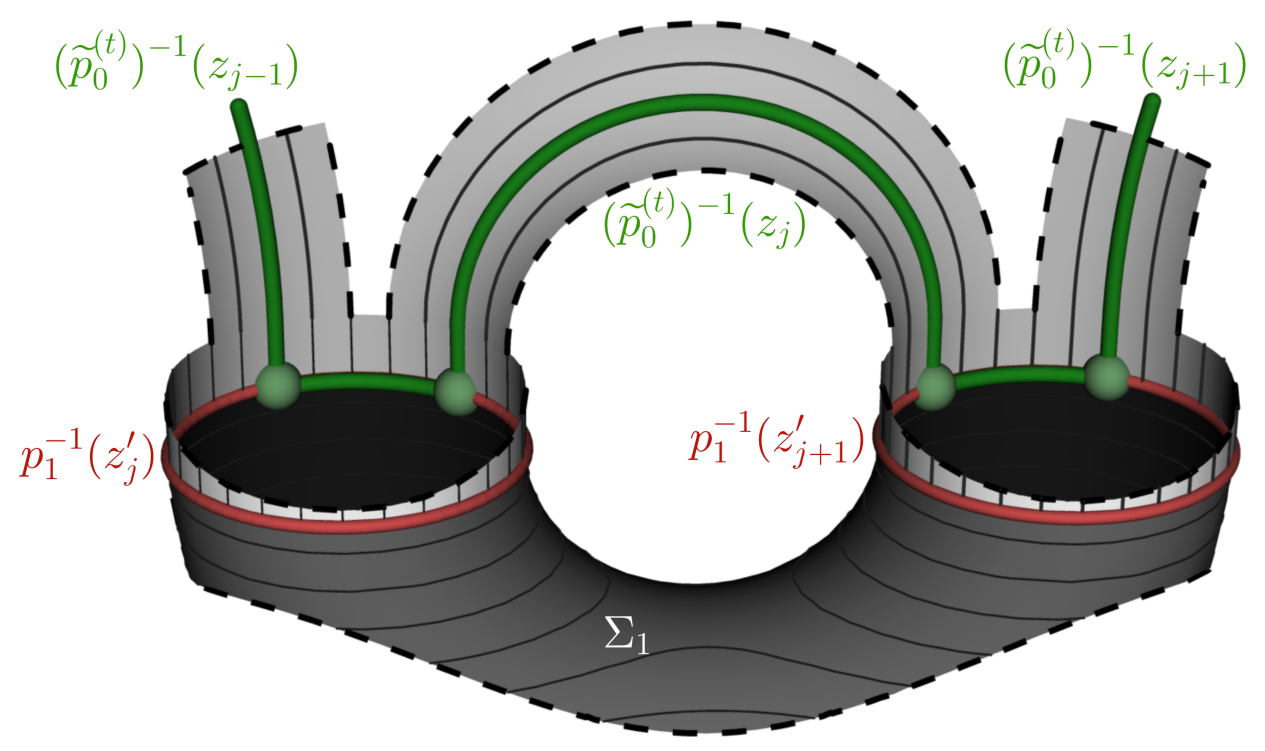

FIGURE 2. Chain of $\stackrel{t}{\approx}$-identifications

\subsection{Parametric interpolation lemma.}

Definition 3.13. Let $\Sigma$ be a topological space, and let $\pi: Z_{K} \rightarrow K$ be a map of polyhedra such that every fiber is a (nonempty and) connected graph. A continuous map $P: K \times \Sigma \rightarrow Z_{K}$ is called a parametric foliation over $K$, or a family of foliations parametrized by $K$, if the composition $\pi \circ P: K \times \Sigma \rightarrow K$ is the projection onto the first factor:

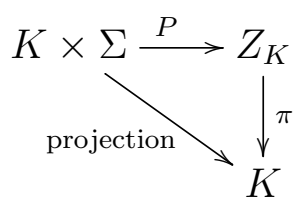

We call $Z_{K}$ the space of leaves, and $\pi$ the parametrization map. For $s \in K$, the restriction $\left.P\right|_{\{s\} \times \Sigma}$ can be viewed as a foliation $p_{s}: \Sigma \rightarrow \pi^{-1}(s)$, and we think of $P$ as the family of foliations $p_{s}$ parametrized by $s \in K$. We say that $P$ is simple if it is PL and connected.

For a parametric foliation $P: K \times \Sigma \rightarrow Z_{K}$ of a metric space $\Sigma$, we keep using the notation $\mathrm{W}(P)=\sup _{z \in Z_{K}} \operatorname{diam} P^{-1}(z)$ for the width.

Definition 3.14. Let $\Sigma$ be a topological space. Let $P_{0}: K \times \Sigma \rightarrow Z_{K}$ be a family of foliations, and let $p_{1}: \Sigma \rightarrow Z_{1}$ be another foliation. An interpolation between them is a parametric foliation $P: C K \times \Sigma \rightarrow Z_{C K}$ over the cone $C K=([0,1] \times K) /(\{1\} \times K)$, restricting to $P_{0}$ over the base $\{0\} \times K$ of $C K$, and to $p_{1}$ over the apex of $C K$.

We are in position to prove the principal lemma of this section.

Lemma 3.15 (Parametric interpolation). Let $\Sigma$ be a metric polyhedron of topological complexity $\beta=\operatorname{tc}(\Sigma)$. Let $P_{K}: K \times \Sigma \rightarrow Z_{K}$ be a family of simple foliations over a d-dimensional complex $K$, and let $p_{1}: \Sigma \rightarrow Z_{1}$ be a simple foliation. It is possible to interpolate between $P_{K}$ and $p_{1}$ via a simple family $C K \times \Sigma \rightarrow Z_{C K}$ of width at most $(\beta+2) \mathrm{W}\left(P_{0}\right)+(\beta+1) \mathrm{W}\left(p_{1}\right)$.

Proof. We can assume $\Sigma$ connected (by dealing with each connected component separately). 
The parametric foliation $P_{K}$ splits into simple foliations $p_{s}: \Sigma \rightarrow Z_{s}$, where $Z_{s}=$ $\pi^{-1}(s), s \in K, \pi: Z_{K} \rightarrow K$ is the parametrization of the foliation base.

The proof idea is simple: for each $s \in K$, interpolate between $p_{s}$ and $p_{1}$ as in Lemma 3.11, and make sure that the interpolation depends nicely on $s$, in order to assemble them altogether to a parametric interpolation. The details are pretty technical, and now we write them out.

The graph $Z_{1}$ is finite and connected, since $\Sigma$ is compact and connected, and $p_{1}$ is simple (hence surjective). Filter $Z_{1}$ as in Lemma 3.12 $Z_{1}^{(0)} \subset \ldots \subset Z_{1}^{(t)} \subset \ldots \subset Z_{1}^{(1)}$, $t \in[0,1]$. We interpolate between $P_{K}$ and $p_{1}$ via a family $P: C K \times \Sigma \rightarrow Z_{C K}$ to be described. With a little abuse of notation, we use coordinates $(t, s) \in[0,1] \times K$ on $C K$, with a convention that all points $(1, s)$ are identified with the apex of $C K$. The restriction $\left.P\right|_{\{(t, s)\} \times \Sigma}$ is a foliation $p_{(t, s)}: \Sigma \rightarrow Z_{(t, s)}$, which can be pictured as follows. First, draw the fibers of $p_{1}$ over $Z_{1}^{(t)}$; then fill in the remaining room with the fibers of $p_{s}$ (with their parts that fit). The resulting picture is interpreted as a foliation by connected leaves, and we call it $p_{(t, s)}$.

We now describe $P: C K \times \Sigma \rightarrow Z_{C K}$ formally.

- Define

$$
\begin{aligned}
& P_{0}: \quad[0,1) \times K \times \Sigma \rightarrow[0,1) \times Z_{K} \\
&(t, s, x) \mapsto\left(t, p_{s}(x)\right) \\
& P_{1}: \quad \rightarrow C \times \Sigma \\
& C K \times Z_{1} \\
&(c, x) \mapsto\left(c, p_{1}(x)\right)
\end{aligned}
$$

- Define

$$
\mathcal{Z}_{1}=\bigcup_{(t, s) \in C K} Z_{1}^{(t)} \subset C K \times Z_{1}
$$

where we think of $Z_{1}^{(t)}$ as sitting in $\{(t, s)\} \times Z_{1}$. The interior of $\mathcal{Z}_{1}$ is

$$
\stackrel{\circ}{\mathcal{Z}}_{1}=\bigcup_{(t, s) \in C K} \stackrel{\circ}{Z}_{1}^{(t)} \subset C K \times Z_{1}
$$

Define

$$
\mathfrak{S}=([0,1) \times K \times \Sigma) \backslash P_{1}^{-1}\left(\stackrel{\circ}{\mathcal{Z}}_{1}\right) \subset[0,1) \times K \times \Sigma
$$

and

$$
\mathcal{Z}_{0}=P_{0}(\mathfrak{S}) \subset[0,1) \times Z_{K}
$$

- The map $\left.P_{0}\right|_{\mathfrak{s}}$ might not be connected, so we factor it through its associated connected map:

$$
\mathfrak{S} \stackrel{\widetilde{P}_{0}}{\rightarrow} \widetilde{\mathcal{Z}_{0}} \rightarrow \mathcal{Z}_{0}
$$

- The space of leaves is

$$
Z_{C K}=\left(\widetilde{\mathcal{Z}_{0}} \sqcup \mathcal{Z}_{1}\right) / \sim
$$

where $\sim$ is the following equivalence relation. Let us write $z \approx z^{\prime}$ if $z \in \widetilde{\mathcal{Z}_{0}}, z^{\prime} \in$ $\mathcal{Z}_{1}$, and $\widetilde{P}_{0}^{-1}(z)$ intersects $P_{1}^{-1}\left(z^{\prime}\right)$, as subsets of $C K \times \Sigma$. (Recall our convention for coordinates in a cone, in which $[0,1) \times K \subset C K$.) Define $\sim$ to be the transitive closure of $\approx$. There are natural maps $\iota_{0}: \widetilde{\mathcal{Z}_{0}} \rightarrow Z_{C K}$ and $\iota_{1}: \mathcal{Z}_{1} \rightarrow Z_{C K}$. 
- The parametric foliation $P$ is defined as

$$
\begin{aligned}
P: C K \times \Sigma & \rightarrow Z_{C K} \\
\xi & \mapsto \begin{cases}\iota_{1}\left(P_{1}(\xi)\right), & \text { if } P_{1}(\xi) \in \mathcal{Z}_{1} \\
\iota_{0}\left(\widetilde{P}_{0}(\xi)\right), & \text { otherwise. }\end{cases}
\end{aligned}
$$

It is easy to see that $P$ indeed interpolates between $P_{K}$ and $p_{1}$.

Clearly, $P$ is connected. It is rather technical but straightforward to make sure that $P$ is $\mathrm{PL}$.

The analysis of the width was already done in Lemma 3.11. Any foliation from the family $P$ interpolates between certain $p_{s}, s \in K$, and $p_{1}$, as in the construction of Lemma 3.11. Therefore, $\mathrm{W}(P) \leq(\beta+2) \mathrm{W}\left(P_{0}\right)+(\beta+1) \mathrm{W}\left(p_{1}\right)$.

3.6. Waist of a PL map. Finally, we are ready to prove the main theorem of this section.

Theorem 3.16. Let $f: X \rightarrow Y^{m}$ be a PL map from a metric polyhedron $X$ to an $m$-dimensional polyhedron $Y$. Let $\beta=\operatorname{tc}(f)$ be its topological complexity, that is, $\beta=$ $\sup _{y \in Y} \operatorname{tc}\left(f^{-1}(y)\right)$. Then there is a fiber $X_{y}=f^{-1}(y)$ of Urysohn width $y \in Y$

$$
\mathrm{UW}_{1}\left(X_{y}\right) \geq c(m, \beta) \mathrm{UW}_{m+1}(X),
$$

for some positive constant $c$ depending only on $m$ and $\beta$.

Proof. Replacing $f$ with its associated connected map, we can assume that $f$ is connected. Even if $f$ is not a fiber bundle, still locally this is almost the case by Lemma 3.4. For each simplex $\triangle \subset Y$ (of any dimension) in a fine triangulation of $Y$, the map $f$ can be "almost" trivialized over $\triangle$ via a PL map

$$
\Psi_{\triangle}: \triangle \times \Sigma_{\triangle} \rightarrow X_{\triangle}
$$

for some polyhedron $\Sigma_{\triangle}$; this map is a genuine trivialization over $\triangle$, the relative interior of $\triangle$. For each $y \in \triangle$, this map induces the distance function $d_{y}^{\triangle}$ on $\Sigma_{\triangle}$ defined as

$$
d_{y}^{\triangle}\left(x, x^{\prime}\right)=\operatorname{dist}_{X}\left(\Psi_{\triangle}(y, x), \Psi_{\triangle}\left(y, x^{\prime}\right)\right) .
$$

Refining the triangulation of $Y$ if needed, we can assume that all metrics $d_{y}^{\triangle}$ over $y \in \triangle$ are $\varepsilon$-close to one another in the following sense: the "layers" $\Psi_{\triangle}(\triangle \times\{x\})$ have diameter less than $\varepsilon / 2$ for all $x \in \Sigma_{\triangle}$, hence for any $x, x^{\prime} \in \Sigma_{\triangle}$ and any $y, y^{\prime} \in \triangle^{\circ}$ we have $\left|d_{y}^{\triangle}\left(x, x^{\prime}\right)-d_{y^{\prime}}^{\triangle}\left(x, x^{\prime}\right)\right| \leq \varepsilon$.

Suppose that $\mathrm{UW}_{1}\left(X_{y}\right)<w_{0}$, for all $y \in Y$, with $w_{0}=c(m, \beta) \mathrm{UW}_{d+1}(X)$ to be specified later. We get a foliation of $X_{y}$ of width less than $w_{0}$, which can be assumed simple by Lemma 3.9. The idea of the proof is to pick a dense discrete set of points in $Y$, and use those foliations to build a map $F: X \rightarrow Z^{m+1}$ of controlled width. This is done inductively on skeleta of $Y$.

At the zeroth step, for each vertex $v$ of $Y$, pick a simple foliation $F_{v}: X_{v} \rightarrow Z_{v}$ of width less than $w_{0}$.

At the $k^{\text {th }}$ step, $1 \leq k \leq m$, we assume that we already defined $F_{k-1}: X_{Y^{(k-1)}} \rightarrow$ $Z_{Y^{(k-1)}}$, over the $(k-1)$-skeleton of $Y$, of width less than $w_{k-1}$, and we need to extend it over $Y^{(k)}$. Take a $k$-simplex $\triangle \subset Y$, and consider the corresponding "trivialization" $\Psi_{\triangle}: \triangle \times \Sigma_{\triangle} \rightarrow X_{\triangle}$. Pick a point $y$ in the relative interior of $\triangle$, and a simple foliation 
$p_{y}$ of $\Sigma_{\triangle}$ of $d_{y}^{\triangle}$-width $<w_{0}$. We would like to use Lemma 3.15 to build a parametric foliation $P_{\triangle}: \triangle \times \Sigma_{\triangle} \rightarrow Z_{\triangle}$ interpolating between $p_{y}$ and the family of foliations

$$
\partial \triangle \times \Sigma_{\triangle} \stackrel{\Psi_{\triangle}}{\rightarrow} X_{\partial \triangle} \stackrel{F_{k-1}}{\rightarrow} Z_{Y^{(k-1)}}
$$

(here $\partial$ denotes the relative boundary). In order to apply that lemma, we need to fix a metric on $\Sigma_{\triangle}$, so we use $d_{y}^{\triangle}$ (recall that they are all $\varepsilon$-close). We get a map $P_{\triangle}: \triangle \times \Sigma_{\triangle} \rightarrow$ $Z_{\triangle}$ of width less than $(\beta+2) w_{k-1}+(\beta+1) w_{0}$. The desired map $F_{\triangle}: X_{\triangle} \rightarrow Z_{\triangle}$ that we are looking for is already defined over $\partial \triangle$, so we specify it over $\triangle$ :

$$
X_{\triangle} \stackrel{\Psi_{\triangle}^{-1}}{\rightarrow} \stackrel{\triangle}{\triangle} \times \Sigma \stackrel{P_{\triangle}}{\rightarrow} Z_{\triangle}
$$

The resulting map $F_{\triangle}$ is continuous. Repeating this over all $k$-simplices we get the map $F_{k}: X_{Y^{(k)}} \rightarrow Z_{Y^{(k)}}$ of width less than

$$
w_{k}=(\beta+2) w_{k-1}+(\beta+1) w_{0}+\varepsilon .
$$

As $\varepsilon \rightarrow 0$, the solution of this recurrence tends to

$$
w_{k}=\left(2(\beta+2)^{k}-1\right) w_{0} .
$$

Therefore, $\mathrm{UW}_{m+1}(X) \leq\left(2(\beta+2)^{m}-1\right) c(m, \beta) \mathrm{UW}_{m+1}(X)$. Hence, for each $c<$ $\frac{1}{2(\beta+2)^{m}-1}$, there is a fiber $X_{y(c)}$ of width at least $c \mathrm{UW}_{m+1}(X)$. Finally, send $c \rightarrow \frac{1}{2(\beta+2)^{m}-1}$, pick a limit point $\bar{y}$ of $\{y(c)\}$, and note that $\mathrm{UW}_{1}\left(X_{\bar{y}}\right) \geq \frac{\mathrm{UW}_{m+1}(X)}{2(\beta+2)^{m}-1}$ by upper semicontinuity of width (Lemma 1.2).

This proof gives the value $c=\frac{1}{2(\beta+2)^{m}-1}$. A more careful analysis of the proof leads to a much better value, namely $c=\frac{1}{2 \beta m+m^{2}+m+1}$, which we explain now.

The inductive interpolation step in the proof of Theorem 3.16 is done in a manner that allows us to split $Y$ into $m$-simplices (basically according to the barycentric subdivision of the triangulation used in induction), so that over each simplex $\triangle$ we have the following picture. Over the vertices of $\triangle$, we have simple foliations $p_{j}: \Sigma_{\triangle} \rightarrow Z_{j}, j=0,1, \ldots, m$. Over a generic point of $\triangle$, we have a foliation $p: \Sigma_{\triangle} \rightarrow Z$ that looks as follows. First, draw the fibers of $p_{m}$ over $Z_{m}^{\left(t_{m}\right)}$, a subgraph of $Z_{m}$ (connected or empty). In the remaining room, draw (the parts of) the fibers of $p_{m-1}$ over $Z_{m-1}^{\left(t_{m-1}\right)}$, a subgraph of $Z_{m-1}$. Continue in the same fashion. At the last step, fill in the remaining room with (the parts of) the fibers of $p_{0}$. The touching fibers of different $p_{j}$ get merged to a single fiber of $p$. What is the maximal length of a chain of merged fibers? We show that it can be bounded by $2 \beta m+m^{2}+m+1$.

Lemma 3.17. Let $\Sigma$ be a metric polyhedron of topological complexity $\beta=\operatorname{tc}(\Sigma)$. Let $p_{j}: \Sigma \rightarrow Z_{j}, j=0,1, \ldots, m$, be simple foliations of width at most 1 . Suppose a parametric foliation $P: \triangle \times \Sigma \rightarrow Z_{\triangle}$ over an m-simplex (restricting to $p_{j}$ over the $j^{\text {th }}$ vertex of $\triangle$ ) is obtained by applying Lemma 3.15 inductively; that is, one first interpolates between $p_{0}$ and $p_{1}$, then between the result and $p_{2}$, and so on. Then the width of $P$ is at most $2 \beta m+m^{2}+m+1$.

Proof. As explained above, a generic foliation $p$ in the family $P$ is obtained by drawing fibers of $p_{j}$ over $Z_{j}^{\left(t_{j}\right)}, j=0,1, \ldots, m$. We assume that every $Z_{j}^{\left(t_{j}\right)}$ is non-empty, otherwise the result follows by induction on $m$. Denote by $\Sigma_{j}$ the closed subset of $\Sigma$ covered (in the foliation $p$ ) by the fibers of $p_{j}, \ldots, p_{m}$ (in particular, $\Sigma_{0}=\Sigma$ ). Notice that for $1 \leq j \leq m$, $\Sigma_{j}$ consists of at most $m-j+1$ connected components, since each set $p_{j}^{-1}\left(Z_{j}^{\left(t_{j}\right)}\right)$ is connected by Lemma 3.6. From the long exact sequence

$$
\ldots \rightarrow H_{1}(\Sigma) \rightarrow H_{1}\left(\Sigma, \Sigma_{j}\right) \rightarrow \tilde{H}_{0}\left(\Sigma_{j}\right) \rightarrow \ldots
$$


one finds that rk $H_{1}\left(\Sigma, \Sigma_{j}\right) \leq \operatorname{rk} H_{1}(\Sigma)+\operatorname{rk} \tilde{H}_{0}\left(\Sigma_{j}\right) \leq \beta+m-j$.

We need to bound the number of fibers in a merged chain. Fix two points $x, y \in \Sigma$ in a single fiber $p^{-1}(z)$, and connect them by a path $\alpha:[0,1] \rightarrow \Sigma$ inside this fiber. For each $t$, notice which of the regions $\Sigma_{j} \backslash \Sigma_{j+1}$ the point $\alpha(t)$ belongs to, and write down the corresponding index $J(t)$ (here $\Sigma_{m+1}$ is assumed empty). We have a piecewise constant function $J:[0,1] \rightarrow\{0,1, \ldots, m\}$. Denote the number of its discontinuities by $D$; without loss of generality, $D$ is finite. Note that $\operatorname{dist}(x, y) \leq D+1$. We will transform $\alpha$ (while keeping it inside the same fiber of $p$, and fixing its endpoints $x, y$ ) to achieve $D \leq(2 \beta+m+1) m$. Consider the following property, which $\alpha$ may or may not enjoy.

Desired property. For $1 \leq j \leq m$, we say that a path $\alpha$ is $j$-nice if the superlevel set $I^{\geq j}=\{t \in[0,1] \mid J(t) \geq j\}$ consists of at most $\beta+m-j+1$ components. We say that $\alpha$ is nice if it $j$-nice for all $1 \leq j \leq m$.

Suppose first $\alpha$ is not nice, and take the smallest index $j$ such that $\alpha$ is not $j$-nice. Mark a point in each component of $I^{\geq j}$, so that we have marked points $t_{1}, \ldots, t_{k}, k>$ $\beta+m-j+1$. Each arc $\alpha\left(\left[t_{i}, t_{i+1}\right]\right)$ represents an element of $H_{1}\left(\Sigma, \Sigma_{j}\right)$. Recall that rk $H_{1}\left(\Sigma, \Sigma_{j}\right) \leq \beta+m-j$. It follows that some two points $\alpha\left(t_{i}\right), \alpha\left(t_{i^{\prime}}\right)$ can be connected inside $p^{-1}(z) \cap \Sigma_{j}$. Replace $\alpha\left(\left[t_{i}, t_{i^{\prime}}\right]\right)$ with this new curve. We decreased the number of components of $I^{\geq j}$. Proceeding in the same fashion, we can make $\alpha j$-nice. Repeating this procedure for larger $j$ if needed, we make $\alpha$ nice.

Now that $\alpha$ is nice, we bound its number $D$ of discontinuities. Clearly, $D$ is bounded by the total number of the endpoints of all $I^{\geq j}$. Since $\alpha$ is nice,

$$
D \leq \sum_{j=1}^{m} 2(\beta+m-j+1)=(2 \beta+m+1) m .
$$

We remark that the improved bound still does not seem sharp. In Gromov's example (example (A) of the introduction) the dependence on $\beta$ is of order $\beta^{-1 / 3}$ while our bound only guarantees $\beta^{-1}$.

Remark 3.18. The proof of Theorem 3.16 together with the estimate of Lemma 3.17 hold with $\mathrm{tc}(\cdot)$ replaced by $\operatorname{tc}^{\prime}(\cdot)$. Indeed, in the proof of Lemma 3.17, to each connected component of $p^{-1}(z) \cap \Sigma_{j}$ that $\alpha$ meets, one can assign a class in $H^{1}(\Sigma)$ in a way so that their products vanish, and their linear dependencies form at most $(m-j+1)$ dimensional space (since they all arise from 0-cochains that are characteristic functions of the connected components of $\Sigma_{j}$ ).

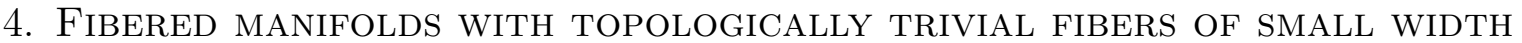

The following result generalizes example (D) from the introduction.

Theorem 4.1. For any non-negative integers $m, k$, and any $\varepsilon>0$, there exists a map $X \rightarrow Y$ such that

- $X=F \times Y$, and the map is the trivial fiber bundle $F \times Y \rightarrow Y$;

- $Y$ and $F$ are closed topological balls of dimensions $m$ and $m k+m+k$, respectively;

- $X$ is endowed with a riemannian metric with $\mathrm{UW}_{n-1}(X) \geq 1$, where $n=\operatorname{dim} X=$ $m k+2 m+k$

- for each $y \in Y$, the fiber $X_{y} \simeq F$ has $\mathrm{UW}_{k+m}\left(X_{y}\right)<\varepsilon$.

Remark 4.2. Consider the trivial bundle $X^{\prime}=F^{\prime} \times Y^{\prime} \rightarrow Y^{\prime}$, where $Y^{\prime}$ is the euclidean $m$-ball of radius $\sim \varepsilon$, and $F^{\prime}$ is the euclidean $(m k+m+k)$-ball of radius $\sim \varepsilon$. The bundle $X$ in the theorem will be constructed in a way so that near its boundary $X$ will 
look exactly like $X^{\prime}$. This allows to modify the construction to make $X$ a closed manifold (e.g., a sphere or a torus), or to take the connected sum with other fibrations, etc.

Proof. To start with, take $Y=\mathbb{R}^{m}, F=\mathbb{R}^{m k+m+k}, X=F \times Y=\mathbb{R}^{m k+2 m+k}$, and ignore for the moment that they are not closed balls. Let $p: X \rightarrow Y$ and $p_{F}: X \rightarrow F$ be the projection maps. We start from the euclidean metric on $X$, modify it, and then cut $X$ to make it compact. Then the (restricted) map $p$ will be the one we are looking for.

On the first factor $F=\mathbb{R}^{m k+m+k}$, consider the structure of the $\varepsilon$-local join of $k$ dimensional complexes $Z_{0}, \ldots, Z_{m}$ in the sense of Definition 2.5. The construction is based on the idea of blowing up the metric in between the $Z_{i}$ (cf. [5, Section 4], where a similar idea is used). Let $\tau: F \rightarrow \triangle^{m}$ be the join map. We think of $\triangle^{m}$ as sitting in $\mathbb{R}^{m}$ with the center at the origin, scaled so that the inradius of $\triangle^{m}$ equals 3 . Consider the "perturbation of the projection via the join map"

$$
p^{\tau}: X \rightarrow Y, \quad p^{\tau}=p-\tau \circ p_{F} .
$$

It will be useful to look at $X$ in the coordinates $\Phi=\left(p_{F}, p^{\tau}\right)$. Namely, $\Phi: X \rightarrow X$ is the map given by $\Phi(x)=\left(p_{F}(x), p^{\tau}(x)\right) \in F \times Y=X$; its inverse is given by $x \mapsto$ $\left(p_{F}(x), p(x)+\tau \circ p_{F}(x)\right)$. It follows that the fibers of $p^{\tau}$ are PL homeomorphic to $F$.

Let $\phi_{1}:[0,+\infty) \rightarrow \mathbb{R}$ be a monotone cut-off function that equals 1 on $[0,1]$ and 0 on $[1.1, \infty)$. Denote by $\phi_{r}^{k}: \mathbb{R}^{k} \rightarrow \mathbb{R}$ an $r$-sized bump function $\phi_{r}^{k}(x):=\phi_{1}(|x| / r)$; here $|\cdot|$ is the euclidean norm in $\mathbb{R}^{k}$. Let $g_{X}^{\text {euc }}, g_{Y}^{\text {euc }}, g_{F}^{\text {euc }}$ be the standard metrics on the corresponding euclidean spaces, viewed as symmetric 2 -forms. To define a new metric on $X$ we take $g_{X}^{\text {euc }}$, blow it up transversely to $\left(p^{\tau}\right)^{-1}(x)$ for $x$ close to the origin of $\mathbb{R}^{m}$, and squeeze everywhere else. Formally,

$$
g_{X}=\Phi^{*} g_{X}^{\prime} \text {, where } g_{X}^{\prime}=\varepsilon g_{X}^{\text {euc }}+(1-\varepsilon)\left(\phi_{2}^{m k+m+k} g_{F}^{\text {euc }}\right) \times\left(\phi_{2}^{m} g_{Y}^{\text {euc }}\right) .
$$

In order for this to be well-defined, one might want to approximate $\Phi$ by a smooth map. From now on, we assume that $X$ is endowed with metric $g_{X}$. To make $X$ compact, one can replace it by its subset $B_{3}^{g_{F}^{\text {euc }}}(0) \times B_{3+m}^{g_{Y}^{\text {euc }}}(0)$. Radius $3+m$ here is chosen so that the 2.2-neighborhood of $\triangle^{m}$ is covered by $p(X)$. We write $X^{\prime}$ for the space $\Phi(X)$ with metric $g_{X}^{\prime}$; clearly, $X$ and $X^{\prime}$ are isometric.

Figure 3 depicts the case $m=1, k=0$ : there, $X=\mathbb{R}^{2}$ is sliced by lines $p^{-1}(y)$ (bold black curves in the figure), each of which is the local join of a green point set $Z_{0}$ and a blue point set $Z_{1}$. On the left, the geometry of $g_{X}$ is depicted by stretching $X$ along the vertical direction, so that it corresponds to the value of $p^{\tau}$. On the right, one sees $X$ in the coordinates $\Phi=\left(p_{f}, p^{\tau}\right)$, with the pinching in the region where $\left|p^{\tau}(x)\right|>2$.

Now let us verify the claimed properties of the metric $g_{X}$. To see that $\mathrm{UW}_{n-1}(X) \geq 1$, note that the unit ball $B_{1}^{g_{X}^{\prime}}(0)$ is just the usual euclidean ball, and its width is $>1$.

Finally, we show that the fibers of $p$ have small width. Consider a fiber $X_{y}=p^{-1}(y), y \in$ $Y$, and the restriction of $g_{X}$ on it. It equals $\varepsilon g_{F}^{\text {euc }}$ plus a term supported in $\tau^{-1}\left(B_{2.2}^{g_{Y}^{\text {euc }}}(y)\right)$. The ball $B_{2.2}^{g_{Y}^{\text {euc }}}(y)$ does not reach one of the faces $v_{i}^{\vee}$ of $\triangle^{m}$. We would like to use the retraction $\pi_{i}$ (as in the discussion after Definition 2.5) to map $p^{-1}(y)$ to $Z_{i}$; this is not possible for the points in the dual complex $Z_{i}^{\vee}$, which is entirely contained in the squeezed zone, so we will not lose much if we just send it to a single point. Here is the map witnessing $\mathrm{UW}_{k+m}\left(X_{y}\right) \lesssim \varepsilon:$

$$
\begin{aligned}
X_{y} \simeq F & \rightarrow\left(Z_{i} \times \triangle^{m}\right) /\left(Z_{i} \times v_{i}^{\vee}\right) \\
x & \mapsto \begin{cases}\left(\pi_{i}(x), \tau(x)\right), & \text { if } x \notin Z_{i}^{\vee} \\
\star, & \text { otherwise. }\end{cases}
\end{aligned}
$$




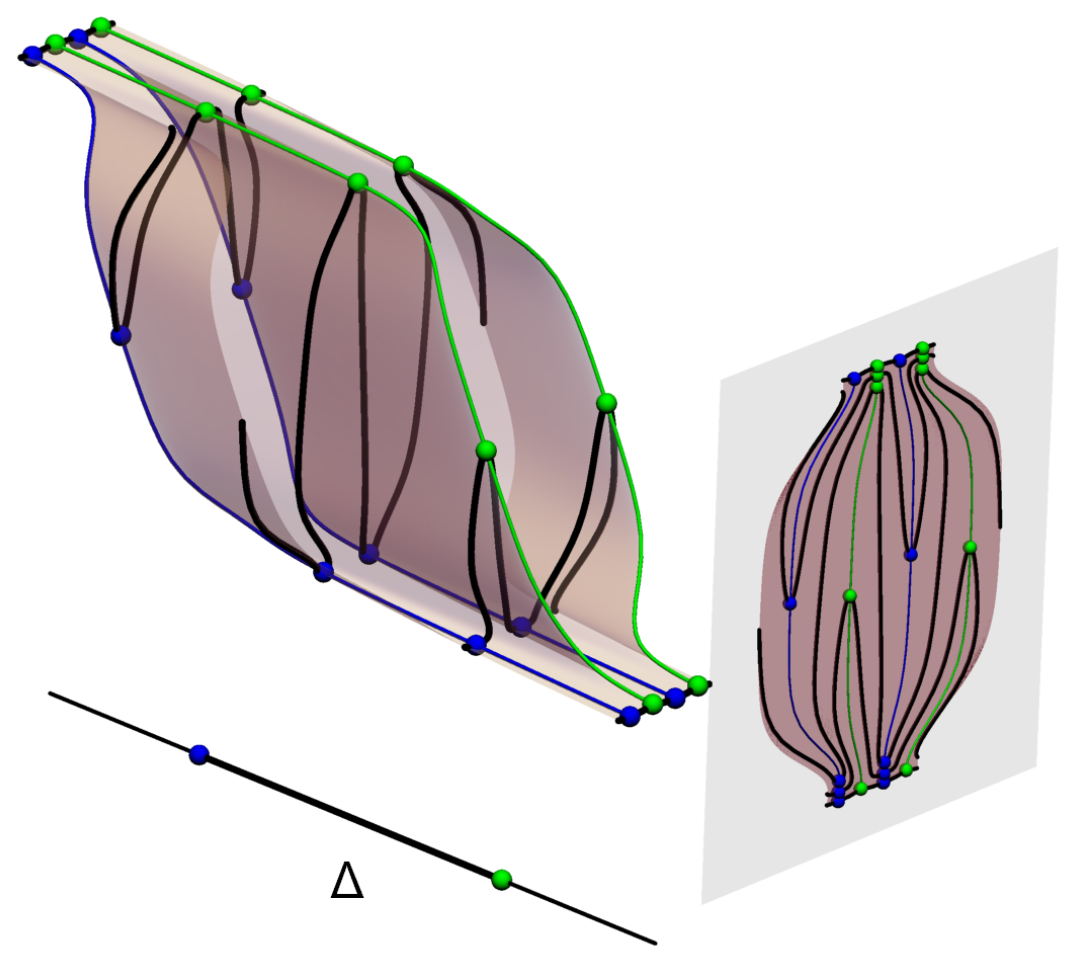

Figure 3. On the left: the map $p: X \rightarrow Y$, with $X$ stretched vertically according to the values of $p^{\tau}$. On the right: $X$ viewed in the coordinates $\left(p_{f}, p^{\tau}\right)$

where $\star$ denotes the pinched copy of $Z_{i} \times v_{i}^{\vee}$ in the quotient. The fiber of this map over $\star$ is $\varepsilon$-small since the metric is squeezed around $Z_{i}^{\vee}$. Consider the fiber over any other point $(z, t)$ of the quotient; since it is contained in $\tau^{-1}(t)$, its $g_{X}$-size does not exceed its $g_{F}$-size; since it is contained in $\pi_{i}^{-1}(z)$, its $g_{F}$-size is $\varepsilon$-small.

\section{REFERENCES}

[1] A. Akopyan, A. Hubard, R. Karasev, et al. Lower and upper bounds for the waists of different spaces. Topological Methods in Nonlinear Analysis, 53(2):457-490, 2019.

[2] A. Akopyan, R. Karasev, and A. Volovikov. Borsuk-Ulam type theorems for metric spaces. arXiv preprint arXiv:1209.1249, 2012.

[3] P. Alexandroff. Notes supplémentaires au "Mémoire sur les multiplicités Cantoriennes", rédigées d'après les papiers posthumes de Paul Urysohn. Fundamenta Mathematicae, 1(8):352-359, 1926.

[4] F. Balacheff. Measurements of Riemannian two-disks and two-spheres. Pacific Journal of Mathematics, 275(1):167-181, 2015.

[5] A. Balitskiy and A. Berdnikov. Local-to-global Urysohn width estimates. arXiv preprint arXiv:2008.07718, 2020.

[6] R. Balka. Dimensions of fibers of generic continuous maps. Monatshefte für Mathematik, 184(3):339$378,2017$.

[7] L. E. J. Brouwer. Über den natürlichen Dimensionsbegriff. Journal für die reine und angewandte Mathematik, 142:146-152, 1913.

[8] M. Gromov. Filling Riemannian manifolds. Journal of Differential Geometry, 18(1):1-147, 1983.

[9] M. Gromov. Width and related invariants of Riemannian manifolds. Astérisque, 163-164:93-109, 1988.

[10] M. Gromov. Isoperimetry of waists and concentration of maps. Geometric $\mathcal{E}$ Functional Analysis GAFA, 13(1):178-215, 2003.

[11] L. Guth. Lipshitz maps from surfaces. Geometric \& Functional Analysis GAFA, 15(5):1052-1090, 2005. 
[12] L. Guth. Systolic inequalities and minimal hypersurfaces. Geometric and Functional Analysis, 19(6):1688-1692, 2010.

[13] L. Guth. The waist inequality in Gromov's work. In H. Holden and R. Piene, editors, The Abel Prize 2008-2012, pages 181-195. Springer, Berlin, 2014.

[14] L. Guth. Volumes of balls in Riemannian manifolds and Uryson width. Journal of Topology and Analysis, 9(02):195-219, 2017.

[15] R. Karasev and A. Volovikov. Waist of the sphere for maps to manifolds. Topology and its Applications, 160(13):1592-1602, 2013.

[16] B. Knaster, C. Kuratowski, and S. Mazurkiewicz. Ein Beweis des Fixpunktsatzes für $n$-dimensionale Simplexe. Fundamenta Mathematicae, 14(1):132-137, 1929.

[17] H. Lebesgue. Sur la non-applicabilité de deux domaines appartenant respectivement à des espaces à $n$ et $n+p$ dimensions. Mathematische Annalen, 70(2):166-168, 1911.

[18] Y. Liokumovich. Slicing a 2-sphere. Journal of Topology and Analysis, 6(04):573-590, 2014.

[19] Y. Liokumovich and D. Maximo. Waist inequality for 3-manifolds with positive scalar curvature. arXiv preprint arXiv:2012.12478, 2020.

[20] M. Matdinov. Size of components of a cube coloring. Discrete $\& 3$ Computational Geometry, 50(1):185193, 2013.

[21] A. Nabutovsky. Linear bounds for constants in Gromov's systolic inequality and related results. arXiv preprint arXiv:1909.12225, 2019.

[22] P. Papasoglu. Uryson width and volume. Geometric and Functional Analysis, 30(2):574-587, 2020.

[23] J.-Y. Shi. The Kazhdan-Lusztig cells in certain affine Weyl groups, volume 1179 of Lecture Notes in Mathematics. Springer-Verlag Berlin Heidelberg, 1986.

[24] V. Tikhomirov. Some questions of the approximation theory (in Russian). Moscow, MSU, 1976.

[25] H. Wallman and W. Hurewicz. Dimension theory. Princeton University Press, 112, 1941.

Email address: "balitski@mit.edu

Email address: "aberdnik@mit.edu

+4 Dept. of Mathematics, Massachusetts Institute of Technology, 182 Memorial Dr., CAmbridge, MA 02142, USA

* Institute for Information Transmission Problems RAS, Bolshoy Karetny Per. 19, Moscow, Russia 127994 